AWARD ACCOUNTS SPSJ Award Accounts

\title{
Macromolecular Assemblies in Solution: Characterization by Light Scattering
}

\author{
By Takahiro SATO ${ }^{1, *}$ and Yasuhiro MATSUDA ${ }^{2}$
}

This review article demonstrates that the light scattering technique provides important structural information of the following macromolecular assemblies, polymer living anions in a non-polar solvent, amphiphilic telechelic polymer and block copolymers in aqueous solution, and a thermally denatured double-helical polysaccharide aggregated during renaturation. To get accurate information from light scattering data, however, we have to analyze the data using proper theoretical tools, which are briefly explained in this article. The structural information of these macromolecular assemblies assists us in understanding, e.g., the kinetics of living anionic polymerization and gelation.

KEY WORDS: Macromolecular Assembly / Light Scattering / Micellization / Aggregation / Telechelic Polymer / Block Copolymer /

Helical Polymer /

Associating polymers, bearing functional groups of strong attractive interactions, hydrophobic, solvophobic, electrostatic interactions, or hydrogen-bonding, form macromolecular assemblies in solution. ${ }^{1}$ There are many types of architecture of macromolecular assemblies ( $c f$. Figure 1). For associating polymers with a single attractive moiety per chain (e.g., diblock copolymers, polymer living anions, water-soluble polymers hydrophobically modified at one end), the architecture may be spherical (star-like), cylindrical, bilayer or vesiclar. $^{2-7}$ On the other hand, if the chain possesses a number of attractive moieties, its assembly may be a uni-core or multicore flower micelle. ${ }^{8-13}$ Multi-stranded helical polymers may form assemblies of their unique architecture by the hydrogenbonding. ${ }^{14}$

The macromolecular assemblies formed by associating polymers in solution can be characterized in terms of the architecture, the size (i.e., the radius of gyration, hydrodynamic radius, or intrinsic viscosity), the average and distribution of the aggregation number, and inter-assembly interaction. However, the determination of these characteristics is not necessarily an easy task in comparison with molecularly dispersed polymers.

The difficulty comes from (1) the concentration dependence of the degree of aggregation, (2) the variety and complexity of the architecture, and (3) the dispersity in the assembly structure in the case of open association systems. ${ }^{15}$ Because of (1), the standard procedure of infinite dilution and also size exclusion chromatography does not necessarily provide right information of the assemblies at a given finite concentration. To make proper characterization, we have to take into account the association-dissociation equilibrium and inter-assembly interaction of associating polymers in solution of the finite concentration. To overcome the difficulties (2) and (3), we have to formulate characteristic parameters using models suitable for each architectures considering the dispersity effect if necessary.

The present article reviews recent light scattering studies on various macromolecular assemblies in dilute solution. Light scattering is one of the most suitable techniques to characterize macromolecular assemblies in solution. Its measurements are made on assemblies in solution without any perturbations, and nanometer to sub-micrometer structures can be investigated. However, analyses of light scattering data are accompanied with the above mentioned difficulties. In what follows, we will explain the method for each system to cope with the difficulties.

\section{THEORETICAL}

\section{Association-Dissociation Equilibrium}

Let us consider an association-dissociation equilibrium between unimer $\mathrm{U}$ and $m$-mer $\mathrm{U}_{m}$ in a polymer solution:

$$
m \mathrm{U} \stackrel{K_{m}}{\rightleftharpoons} \mathrm{U}_{m}
$$

The equilibrium constant $K_{m}$ is determined from the condition given by $\mu_{m}=m \mu_{1}$, where $\mu_{m}$ and $\mu_{1}$ are the chemical potentials of $m$-mer and unimer, respectively. From statistical thermodynamics, $\mu_{m}$ can be generally written as ${ }^{16,17}$

$$
\frac{\mu_{m}}{k_{\mathrm{B}} T}=\frac{\mu_{m}^{\circ}}{k_{\mathrm{B}} T}+\ln \left(\frac{w_{m} \phi}{m}\right)+m F_{1}(\phi)+F_{2}(\phi)
$$

where, $\mu_{m}{ }^{\circ}, w_{m}$, and $\phi$ are the standard chemical potential (or the internal free energy), the weight fraction of $m$-mer in the total polymer, and the total volume fraction of the polymer, respectively, $F_{1}(\phi)$ and $F_{2}(\phi)$ are functions of $\phi$, and $k_{\mathrm{B}} T$ is the Boltzmann constant multiplied by the absolute temperature.

\footnotetext{
${ }^{1}$ Department of Macromolecular Science, Osaka University, 1-1 Machikaneyama-cho, Toyonaka 560-0043, Japan

${ }^{2}$ Department of Materials Science and Chemical Engineering, Shizuoka University, 3-5-1 Johoku, Naka-ku, Hamamatsu 432-8561, Japan

*To whom correspondence should be addressed (Tel: +81-6-6850-5461, Fax: +81-6-6850-5461, E-mail: tsato@ chem.sci.osaka-u.ac.jp).
} 


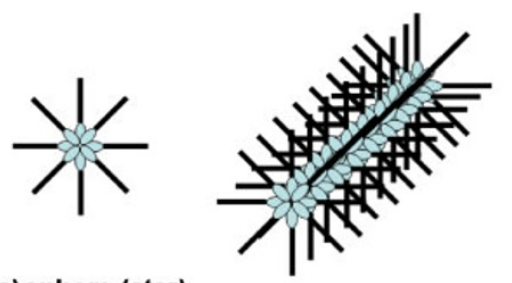

(a)sphere (star)

(b) cylinder

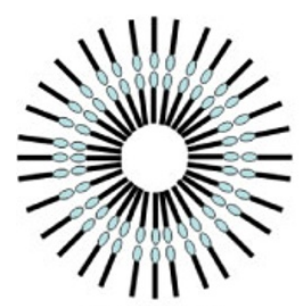

(d) vesicle

(e) flower

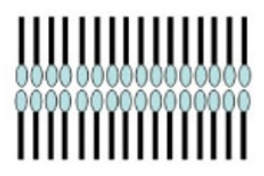

(c) bilayer

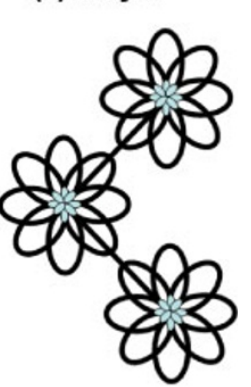

(f) bouquet

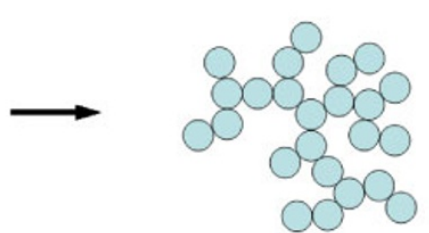

(g) random aggregate

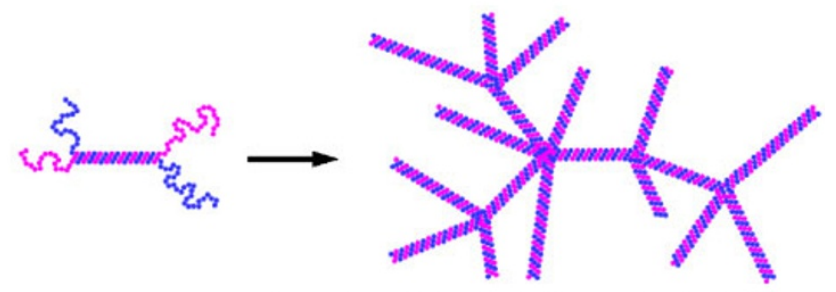

(h) mismatched winding of double helices

Figure 1. Various types of architecture of macromolecular assemblies.

( $\mu_{1}$ can be calculated by eq (1.2) with $m=1$.) Various statistical thermodynamic theories of polymer solutions proposed different functional forms of $F_{1}(\phi)$ and $F_{2}(\phi)$, only the latter of which is important in the equilibrium condition. The Flory-Huggins theory ${ }^{16,18}$ gives us $F_{2}(\phi)=1$, and the scaled particle theory for hard spherocylinders provides $^{17,19}$

$$
F_{2}(\phi)=\frac{5 \phi-3 \phi^{2}}{3(1-\phi)^{2}}-\ln (1-\phi)
$$

Inserting eq (1.1) into the equilibrium condition, we obtain

$$
w_{m}=K_{m} w_{1}{ }^{m} \phi^{m-1}
$$

where $w_{1}$ is the weight fraction of unimer in the solution, which can be determined from the normalization condition, $\sum_{m} w_{m}=1$, and the equilibrium constant $K_{m}$ is given by

$$
K_{m} \equiv m \exp \left[-\Delta_{m}+(m-1) F_{2}(\phi)\right]
$$

where $\Delta_{m} \equiv\left(\mu_{m}{ }^{\circ}-m \mu_{1}{ }^{\circ}\right) / k_{\mathrm{B}} T$ is the free energy of the $m$ mer formation in units of the thermal energy $k_{\mathrm{B}} T$.

The expression of $\Delta_{m}$ depends on the type of association. At the formation of a uni-core star or flower micelle from $m$ single chains, $\Delta_{m}$ may be given by ${ }^{20,21}$

$$
\Delta_{m}=-\delta(\lambda m)+\sigma(\lambda m)^{2}+4 \pi R_{\mathrm{c}}^{2} \gamma
$$

with the number $\lambda$ of solvophobic groups per chain in the micelle core. Here, the first term on the right hand side correspond to the free energy gain when $\lambda m$ solvophobic groups enter in the micelle core from the solvent, the second term the free energy due to interaction among coronal chains in the micelle, and the third term the interfacial free energy of the micelle core; $\delta$ and $\sigma$ are free energy coefficients, $R_{\mathrm{c}}$ the radius of the micellar core, and $\gamma$ the interfacial tension. From eqs (1.4)-(1.6), the optimum aggregation number $m_{0}$ of the micelle is equal to $\left(4 \pi R_{\mathrm{c}}{ }^{2} \gamma / \sigma\right)^{1 / 2}$, and the micellar size distribution is determined by $\sigma$. If $\sigma$ is large enough, the aggregation number can be regarded essentially to be monodisperse $\left(m=m_{0}\right)$. The free energy of the micellar formation $\Delta_{m} / m$ per monomer at $m=m_{0}$ is usually denoted as $\varepsilon_{0}$, which mainly determines the association constant $K_{m}$.

On the other hand, in the case of the random association of $f$-functional unimers, $\Delta_{m}$ may be simply written as ${ }^{16}$

$$
\Delta_{m}=(m-1)\left(\Delta f_{0} / k_{\mathrm{B}} T\right)-\ln W_{m}
$$

where $\Delta f_{0}$ is the free energy change at the single physical bond formation and $W_{m}$ is the number of ways where $m$ unimers form an $m$-mer. It is noted that $m$-mer possesses $m-1$ physical bonds. Under the tree approximation, $W_{m}$ is written as $^{22}$

$$
W_{m}=\frac{f^{m}(f m-m) !}{(f m-2 m+2) !}
$$

Equations (1.4), (1.5), and (1.7) provide us $w_{m}$ and the weight average aggregation number $m_{\mathrm{w}}$ in the forms

$$
w_{m}=\frac{1}{(m-1) !} W_{m}(\alpha / f)^{m-1}(1-\alpha)^{f m-2 m+2}
$$

and

$$
m_{\mathrm{w}}=\frac{1+\alpha}{1-(f-1) \alpha}
$$

Here $\alpha$ is the fraction of physical bonding, which is related to the total polymer volume fraction $\phi$ by

$$
\alpha=\frac{1+2 f X \phi-\sqrt{1+4 f X \phi}}{2 f X \phi}
$$

with $X \equiv \exp \left[F_{2}(\phi)-\Delta f_{0} / k_{\mathrm{B}} T\right]$. The distribution $w_{m}$ is broader for larger $f$ and $m_{\mathrm{w}}$ diverges at $\alpha=1 /(f-1)$ (the gel point).

\section{Radius of Gyration}

The radius of gyration $\left\langle S^{2}\right\rangle^{1 / 2}$ is one of the most important molecular parameters determined by static light scattering and characterizing the structure of macromolecular assemblies. In what follows, we present expressions of $\left\langle S^{2}\right\rangle^{1 / 2}$ for (1) the starlike micelle, (2) the core-corona-type micelle, (3) the vesicle, and (4) the random aggregate. 
The star-like micelle formed by $m$ linear flexible chains, each of which possesses an associating group at one chain end, can be identified by a uniform $m$-flexible-arm star polymer, if the micellar core is negligibly small. The square radius of gyration of the star polymer can be written as:

$$
\left\langle S^{2}\right\rangle_{\text {star }}=\frac{3 m-2}{m^{2}}\left\langle S^{2}\right\rangle_{\text {linear }}
$$

or

$$
\left\langle S^{2}\right\rangle_{\text {star }}=1.94 m^{-4 / 5}\left\langle S^{2}\right\rangle_{\text {linear }}
$$

where $\left\langle S^{2}\right\rangle_{\text {linear }}$ is the square radius of gyration of the linear polymer chain with the same molecular weight as that of the star polymer. Although eq (1.12a) was originally derived for Gaussian star polymers by Zimm and Stockmayer, ${ }^{23}$ its validity even in good solvents was confirmed by many experiments and Monte Carlo simulations $(3 \leq m \leq 18) \cdot{ }^{24,25}$ On the other hand, eq $(1.12 b)$ was proposed by Douglas et al. ${ }^{26}$ to fulfill the Daoud-Cotton scaling theory ${ }^{27}$ with respect to the asymptotic behavior, so that it should be applied at large $m$.

Unfortunately, $\left\langle S^{2}\right\rangle$ for flower micelles has not been formulated. Instead, a flower micelle with $p$ petals or loops has been usually approximated by a star-like micelle with $2 p$ arms, being neglected a slight increase in $\left\langle S^{2}\right\rangle$ by cutting of the loop chains.

If the block copolymer forms a star or core-corona micelle in a selective solvent, the micellar core is not necessarily small, so that we have to take into account the effect of the finite core size on $\left\langle S^{2}\right\rangle$. Consider the core-corona micelle consisting of $m$ block copolymer chains each of which comprises a core chain of the molar mass $M_{\text {core }}$ and a flexible coronal chain of the molar mass $M_{\text {corona }}$. The radius $R_{\text {core }}$ of the core may be calculated by

$$
R_{\text {core }}=\left(3 m M_{\text {core }} / 4 \pi N_{\mathrm{A}} \rho_{\text {core }}\right)^{1 / 3}
$$

where $\rho_{\text {core }}$ is the density of the core. The segment density distribution function $\rho(s)$ in the coronal region of the micelle may be approximated by that $\rho_{\text {star }}(s)$ for a uniform $m$-flexiblearm star polymer with a molecular weight of $M_{\text {star }}$, which may be approximated by the Gaussian distribution ${ }^{28}$

$$
\rho_{\text {star }}(s)=\frac{M_{\text {star }}}{N_{\mathrm{A}}}\left(\frac{3}{2 \pi\left\langle S^{2}\right\rangle_{\text {star }}}\right)^{3 / 2} \exp \left(-\frac{3 s^{2}}{2\left\langle S^{2}\right\rangle_{\text {star }}}\right)
$$

Here, $\left\langle S^{2}\right\rangle_{\text {star }}$ is the square radius of gyration of the star polymer. Integrating eq (1.14) over the coronal region, we have the relation

$$
\begin{aligned}
& \int_{R_{\text {core }}}^{\infty} \rho_{\text {star }}(s) \cdot 4 \pi s^{2} d s \\
& \quad=\frac{2 M_{\text {star }}}{\sqrt{\pi} N_{\mathrm{A}}}\left[\operatorname{erfc}(\alpha)+\alpha \mathrm{e}^{-\alpha^{2}}\right]=\frac{m M_{\text {corona }}}{N_{\mathrm{A}}}
\end{aligned}
$$

where $\alpha^{2} \equiv 3 R_{\text {core }}{ }^{2} / 2\left\langle S^{2}\right\rangle_{\text {star }}$ and $\operatorname{erfc}(x)$ is the error function $\left[\operatorname{erfc}(x) \equiv \int_{x}^{\infty} e^{-t^{2}} d t\right]$. From this equation combined with eq (1.12), we can determine $M_{\text {star }}$ and $\left\langle S^{2}\right\rangle_{\text {star }}$ from $m M_{\text {corona }}$ and $R_{\text {core }}$.

When refractive index increments $v(\equiv \partial n / \partial c)$ of the two block chains are different, the light-scattering radius of gyration $\left\langle S^{2}\right\rangle^{* 1 / 2}$ obtained by static light scattering is not identical with the conventionally defined radius of gyration. From the light scattering theory for copolymers, ${ }^{29}\left\langle S^{2}\right\rangle^{*}$ of the core-corona micelle is given by

$$
\begin{aligned}
\left\langle S^{2}\right\rangle^{*} & =\frac{N_{\mathrm{A}} \int_{0}^{\infty} 4 \pi s^{4} \nu^{2}(s) \rho_{\text {star }}(s) d s}{\bar{v}^{2} m\left(M_{\text {core }}+M_{\text {corona }}\right)} \\
& =\frac{\frac{3}{5} M_{\text {core }} v_{\text {core }}{ }^{2} R_{\text {core }}{ }^{2}+\left(\frac{4 M_{\text {star }}}{3 \sqrt{\pi} m} \alpha^{3} \mathrm{e}^{-\alpha^{2}}+M_{\text {corona }}\right) v_{\text {corona }}{ }^{2}\left\langle S^{2}\right\rangle_{\text {star }}}{\bar{v}^{2}\left(M_{\text {core }}+M_{\text {corona }}\right)}
\end{aligned}
$$

where $v(s), v_{\text {core }}$, and $v_{\text {corona }}$ are refractive index increments at the position $s$, of the core chain, and of the coronal chain, respectively, and $\bar{v}$ is the weight-average refractive index increment of the copolymer sample. It is noted that eq (1.16) along with eqs (1.12) and (1.15) has no adjustable parameters, if we know $M_{\text {core }}, M_{\text {corona }}$, $m, \rho_{\text {core }}$ and the molecular weight dependence of $\left\langle S^{2}\right\rangle_{\text {linear }}$ for the linear coronal chain.

The block copolymer also can form a vesicle in a selective solvent under certain conditions. ${ }^{2-6}$ The vesicle consists of a spherical shell and brushes inside and outside the shell. The thicknesses of the shell and inner and outer brushes are denoted as $a, H_{\mathrm{i}}$, and $H_{\mathrm{o}}$, and the inner and outer radii of the shell as $R \pm a / 2$. According to Milner et al., ${ }^{30}$ the concentration profile of the polymer brush is assume to take a parabolic form. Then the segment radial distribution function $\rho_{\text {vesicle }}(s)$ may be written as

$$
\rho_{\text {vesicle }}(s)= \begin{cases}0 & \left(s \leq R_{\mathrm{i}}\right) \\ A_{\mathrm{i}}\left[H_{\mathrm{i}}{ }^{2}-\left(R^{\prime}-s\right)^{2}\right] & \left(R_{\mathrm{i}}<s<R^{\prime}\right) \\ \rho_{\text {shell }} & \left(R^{\prime} \leq s \leq R^{\prime \prime}\right) \\ A_{\mathrm{o}}\left[H_{\mathrm{o}}{ }^{2}-\left(s-R^{\prime \prime}\right)^{2}\right] & \left(R^{\prime \prime}<s<R_{\mathrm{o}}\right) \\ 0 & \left(R_{\mathrm{o}} \leq s\right)\end{cases}
$$

where $\rho_{\text {shell }}$ is the density in the shell region, and

$$
\begin{aligned}
R_{\mathrm{i}} & \equiv R-\frac{1}{2} a-H_{\mathrm{i}}, \quad R^{\prime} \equiv R-\frac{1}{2} a, \\
R^{\prime \prime} & \equiv R+\frac{1}{2} a, \quad R_{\mathrm{o}} \equiv R+\frac{1}{2} a+H_{\mathrm{o}}
\end{aligned}
$$

Furthermore, according to the theory of polymer brushes, ${ }^{30}$ we have the relations

$$
H_{\mathrm{i}}=\kappa \frac{m_{\mathrm{i}}{ }^{1 / 3} M_{\mathrm{brush}}}{R^{\prime 2 / 3}}, \quad H_{\mathrm{o}}=\kappa \frac{m_{\mathrm{o}}{ }^{1 / 3} M_{\mathrm{brush}}}{R^{\prime 2 / 3}}
$$


where $\kappa$ is a constant, and $m_{\mathrm{i}}$ and $m_{\mathrm{o}}$ are the numbers of chains in the inner and outer brushes, respectively.

The two constants $A_{\mathrm{i}}$ and $A_{\mathrm{o}}$ in eq (1.17) are related to the molar mass $M_{\text {brush }}$ of the brush chain by the following equations

$$
\left\{\begin{array}{l}
\int_{R_{\mathrm{i}}}^{R^{\prime}} 4 \pi s^{2} \rho_{\text {vesicle }}(s) d s=4 \pi A_{\mathrm{i}}\left(\frac{2}{3} R^{\prime 2}-\frac{1}{2} R^{\prime} H_{\mathrm{i}}+\frac{2}{15} H_{\mathrm{i}}{ }^{2}\right) H_{\mathrm{i}}{ }^{3}=\frac{m_{\mathrm{i}} M_{\text {brush }}}{N_{\mathrm{A}}} \\
\int_{R^{\prime \prime}}^{R_{\mathrm{o}}} 4 \pi s^{2} \rho_{\text {vesicle }}(s) d s=4 \pi A_{\mathrm{o}}\left(\frac{2}{3} R^{\prime \prime 2}+\frac{1}{2} R^{\prime \prime} H_{\mathrm{o}}+\frac{2}{15} H_{\mathrm{o}}{ }^{2}\right) H_{\mathrm{o}}{ }^{3}=\frac{m_{\mathrm{o}} M_{\text {brush }}}{N_{\mathrm{A}}}
\end{array}\right.
$$

On the other hand, the radius $R$ is related to the molar mass $M_{\text {shell }}$ of the block chain forming the shell by the equation

$$
4 \pi \rho_{\text {shell }} a\left(R^{2}+\frac{1}{12} a^{2}\right)=\frac{\left(m_{\mathrm{i}}+m_{\mathrm{o}}\right) M_{\text {shell }}}{N_{\mathrm{A}}}
$$

The light-scattering radius of gyration $\left\langle S^{2}\right\rangle^{* 1 / 2}$ of the vesicle is given by

$$
\left\langle S^{2}\right\rangle^{*}=\frac{\left[\begin{array}{l}
v_{\text {brush }}{ }^{2} A_{\mathrm{i}} H_{\mathrm{i}}{ }^{3}\left(\frac{2}{3} R^{\prime 4}-R^{\prime 3} H_{\mathrm{i}}+\frac{4}{5} R^{\prime 2} H_{\mathrm{i}}{ }^{2}-\frac{1}{3} R^{\prime} H_{\mathrm{i}}{ }^{3}+\frac{2}{35} H_{\mathrm{i}}{ }^{4}\right) \\
+\frac{1}{5} v_{\text {shell }}{ }^{2} \rho_{\text {shell }}\left(R^{\prime \prime}-R^{\prime 5}\right) \\
+v_{\text {brush }}{ }^{2} A_{\mathrm{o}} H_{\mathrm{o}}{ }^{3}\left(\frac{2}{3} R^{\prime \prime 4}+R^{\prime \prime 3} H_{\mathrm{o}}+\frac{4}{5} R^{\prime \prime 2} H_{\mathrm{o}}{ }^{2}+\frac{1}{3} R^{\prime \prime} H_{\mathrm{o}}{ }^{3}+\frac{2}{35} H_{\mathrm{o}}{ }^{4}\right)
\end{array}\right]}{\bar{v}^{2}\left(m_{\mathrm{i}}+m_{\mathrm{o}}\right)\left(M_{\text {shell }}+M_{\text {brush }}\right) / 4 \pi N_{\mathrm{A}}}
$$

where $\nu_{\text {brush }}$ and $v_{\text {shell }}$ are refractive index increments of the brush and shell chains, respectively.

At last, we consider open association systems where $f$-functional units randomly aggregate according to the formula:

$$
\mathrm{U}_{m}+\mathrm{U}_{n} \rightarrow \mathrm{U}_{m+n} \quad(m, n=1,2, \cdots)
$$

Similar open association systems have been already treated in the previous subsection, but here the systems are in the unidirectional process with functional groups keeping equal reactivity throughout the process. In such cases, the distribution law $w_{m}$ and the weight average aggregation number $m_{\mathrm{w}}$ are given by eqs (1.9) and (1.10), ${ }^{22}$ even if the system is not thermally equilibrium. Furthermore, the cascade theory ${ }^{9,31}$ gives us the following expression of the $\mathrm{z}$-average square radius of gyration

$$
\left\langle S^{2}\right\rangle_{\mathrm{z}}=\frac{b^{2} f \alpha}{2(1+\alpha)^{2}} m_{\mathrm{w}}+\left\langle S^{2}\right\rangle_{\mathrm{u}}
$$

where $b$ is the distance between associating nearest neighbor units, $\alpha$ is the reacted fraction of functional groups, and $\left\langle S^{2}\right\rangle_{\mathrm{u}}$ is the square radius of gyration of the associating unit.

If the above aggregation process is stopped at a certain $\alpha$, and aggregates formed are fractionated according to the aggregation number, the radius of gyration of the fraction with the aggregation number $m$ can be calculated by ${ }^{32}$

$$
\begin{aligned}
\left\langle S^{2}\right\rangle_{m}= & \frac{b^{2}}{2} \frac{(m-2) !}{[(f-1) m] !} \sum_{n=0}^{m-2}(f-1)^{n} \\
& \times \frac{[(f-1) m-n-1] !}{(m-n-2) !}[n(f-2)+2(f-1)] \\
& \times(n+1)+\left\langle S^{2}\right\rangle_{\mathrm{u}}
\end{aligned}
$$

\section{POLYMER LIVING ANIONS IN A NON-POLAR SOLVENT}

Polymer living anions possessing the non-polar main chain and polar active end may form reversed micelles during the living anionic polymerization carried out in non-polar solvents. Micellization plays an important role in polymerization reaction kinetics, because only dissociated free living anions are believed to be active species for the propagation reaction. ${ }^{33-37}$ Thus the precise aggregation number $m$, the association constant (or the free energy of micellar formation), and the structure of the reversed micelle formed by polymer living anions are prerequisite to the profound understanding of the anionic polymerization.

Matsuda et $a .^{21,38}$ studied on the reversed micelle of polybutadiene (PB) living anions in cyclohexane at $25^{\circ} \mathrm{C}$ by light scattering. Light scattering is a convenient method of investigating moisture-sensitive PB living anions, because the measurements can be made on test solutions in sealed glass cells.

Figure 2 shows the concentration $c$ dependences of $\left(K c M_{1} /\right.$ $\left.R_{0}\right)^{1 / 2}$ for the major components of three living polybutadiene (LPB) samples investigated with the molecular weights $M_{1}$ of 9,600 (LPB-10k), 35,200 (LPB-35k), and 81,900 (LPB-82k). Here, $K$ is the optical constant, $c$ is the polymer mass concentration, $M_{1}$ is the molecular weight of PB sample after quench, and $R_{0}$ is the excess Rayleigh ratio at the zero scattering angle. For all three LPB samples, data points obey slightly concave curves, and the intercepts of the curves seem to be less than unity, indicating that LPB aggregates in cyclohexane.

The strong $c$ dependences however arise from the virial terms because cyclohexane is a good solvent for $\mathrm{PB}$, and we 


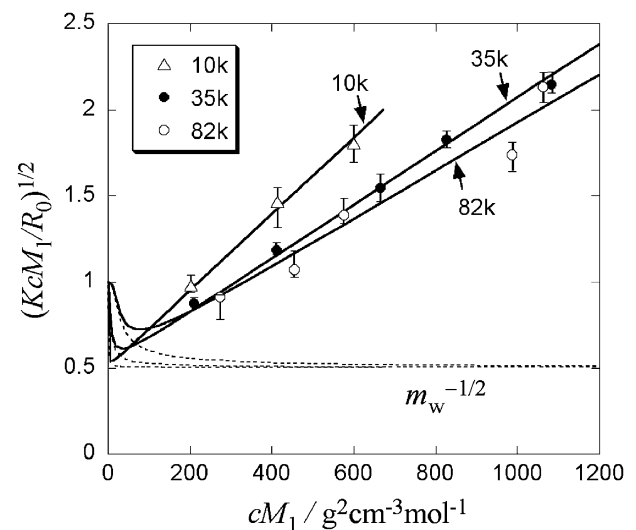

Figure 2. Static light scattering results for three polybutadiene living anion samples in cyclohexane; solid curves, theoretical values calculated by eqs (2.1)-(2.3) along with eq (1.4); dotted curves, $m_{\mathrm{w}}{ }^{-1 / 2}$ calculated by the same equations with the zero virial coefficients. Reproduced with permission from ref 21. Copyright (2007) American Chemical Society.

have to analyze the light scattering data considering the contribution of the virial terms. Using the third virial approximation, we have ${ }^{28}$

$$
\begin{aligned}
\frac{K c M_{1}}{R_{0}}= & \frac{1}{m_{\mathrm{w}}}+2 A_{2, \mathrm{z}}\left(M_{1} c\right) \\
& +\left[\frac{2 A_{3, \mathrm{z}}+A_{3, \mathrm{w}}}{M_{1}}-m_{\mathrm{w}}\left(\overline{A_{2 \mathrm{z}}^{2}}-A_{2, \mathrm{z}}{ }^{2}\right)\right]\left(M_{1} c\right)^{2}
\end{aligned}
$$

where $m_{\mathrm{w}}$ is the weight average aggregation number ( $=\sum_{m \geq 1} m w_{m}$ ), and $A_{2, \mathrm{z}}, \overline{A_{2 \mathrm{z}}^{2}}, A_{3, \mathrm{z}}$, and $A_{3, \mathrm{w}}$ are average second and third virial coefficients defined by

$$
\left\{\begin{array}{l}
A_{2, \mathrm{z}} \equiv m_{\mathrm{w}}{ }^{-1} \sum_{m \geq 1} m w_{m} A_{m m}, \quad \overline{A_{2 \mathrm{z}}^{2}} \equiv m_{\mathrm{w}}{ }^{-1} \sum_{m \geq 1} m w_{m} A_{m m}{ }^{2} \\
A_{3, \mathrm{z}} \equiv m_{\mathrm{w}}{ }^{-1} \sum_{m \geq 1} m w_{m} A_{m m m},
\end{array}\right.
$$

with the second virial coefficient $A_{m m}$ and the third virial coefficient $A_{m m m}$ of $m$-mer.

As demonstrated below, the LPB aggregate is a reversed star micelle, and $A_{m m}$ and $A_{m m m}$ of the star micelle (or the star polymer) can be calculated from the corresponding coefficients $A_{2, m}$ (linear) and $A_{3, m}$ (linear) for the linear chain with the molar mass $m M_{1}$ using relations $A_{m m}=g_{\mathrm{A} 2, m} A_{2, m}$ (linear) and $A_{m m m}=g_{\mathrm{A} 3, m} A_{3, m}$ (linear). From literature data, ${ }^{26,39,40}$ we have the following empirical equations of the $g$-factors for flexible star polymers in good solvents: $g_{\mathrm{A} 2, m}=1-0.04(m-2)$ and $g_{\mathrm{A} 3, m}=1-0.08(m-2)$. Furthermore, we can write $A_{3, m}$ (linear) $=\gamma A_{2, m}(\text { linear })^{2} M_{m}$ where $\gamma$ is empirically known to be 0.3 for linear flexible polymers in good solvents. ${ }^{41}$ Literature data are available for $A_{2, m}$ (linear). ${ }^{42}$ Under the same third virial approximation, eq (1.5) is rewritten in the form

$$
\begin{aligned}
K_{m}= & m \exp \left[-\Delta_{m}+m M_{1}\left(A_{11}-A_{m m}\right) c\right. \\
& \left.+\frac{1}{2} m M_{1}\left(A_{111}-A_{m m m}\right) c^{2}\right]
\end{aligned}
$$

The weight fraction $w_{m}$ of $m$-mer can be calculated

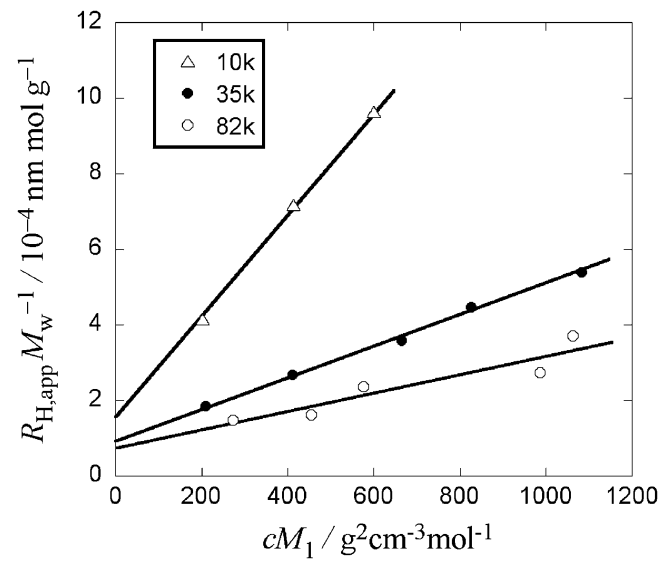

Figure 3. Dynamic light scattering results for three polybutadiene living anion samples in cyclohexane; solid curve, theoretical values calculated by eqs (2.4) and (2.5). Reproduced with permission from ref 21. Copyright (2007) American Chemical Society.

from eqs (1.4) and (2.3) as a function of $\phi$ or $c$ (see THEORETICAL SECTION).

Eqs (2.1)-(2.3) contain two adjustable parameters, the optimum aggregation number $m_{0}$ and the free energy $\varepsilon_{0}$ of the micellar formation per monomer and in units of the thermal energy $k_{\mathrm{B}} T$. (Here, $\sigma$ in eq (1.6) is assumed to be so large that the aggregation number of the micelle is monodisperse.) A best fit to the experimental $\left(K c M_{1} / R_{0}\right)^{1 / 2}$ for LPB micellar solutions was obtained when $m_{0}$ was chosen to be 4 for all three LPB samples. Solid curves in Figure 2 indicate theoretical values calculated from eqs (2.1) and (2.2) using $m_{0}=4$ and $\varepsilon_{0}=-14$, demonstrating good fittings for all three LPB samples. On the other hand, dashed curves in the same figure indicate $\left(K c M_{1} / R_{0}\right)^{1 / 2}$ after subtracting the virial terms, i.e., $1 / m_{\mathrm{w}}{ }^{1 / 2}$. From the results, $m_{\mathrm{w}}$ is so close to 4 in the $c$ range investigated that most of LPB anions form reversed micelles and the dissociation little takes place. In such a $c$ range, theoretical $\left(K c M_{1} / R_{0}\right)^{1 / 2}$ little changes even if we choose a value of $\varepsilon_{0}$ less than -14 , so that the value -14 should be taken as the upper limit of $\varepsilon_{0}$. It was difficult to make light scattering measurements at lower $c$ because of low light scattering intensities.

To investigate the micellar structure of LPB, we have estimated the apparent hydrodynamic radius $R_{\mathrm{H} \text {,app }}$ at finite concentrations divided by the weight-average molar mass $M_{\mathrm{w}}$ for the major component, from the first cumulant $\Gamma$ obtained by dynamic light scattering and $K c / R_{0}$ using the equation $^{43,44}$

$$
\frac{R_{\mathrm{H}, \mathrm{app}}}{M_{\mathrm{w}}}=\frac{k_{\mathrm{B}} T(1-\bar{v} c)}{6 \pi \eta_{0}} \frac{K c / R_{0}}{\lim _{k \rightarrow 0} \Gamma / k^{2}}
$$

where $\bar{v}$ is the partial specific volume, $\eta_{0}$ is the solvent viscosity coefficient, and $k$ is the magnitude of the scattering vector. Figure 3 shows $R_{\mathrm{H} \text {,app }} / M_{\mathrm{W}}$ for cyclohexane solutions of the three LPB samples plotted against $c M_{1}$. The strong $c$ dependences come from the hydrodynamic interaction among polymers. 
For micellar solutions of finite concentrations, $R_{\mathrm{H}, \mathrm{app}} / M_{\mathrm{W}}$ may be calculated by ${ }^{21}$

$$
\frac{R_{\mathrm{H}, \mathrm{app}}}{M_{\mathrm{w}}}=\left(1+k_{\mathrm{H}}^{\prime} c / c^{*}\right) /\left(\frac{w_{1} M_{1}}{R_{\mathrm{H}, 1}}+\frac{w_{m_{0}} M_{m_{0}}}{R_{\mathrm{H}, m_{0}}}\right)
$$

where $k_{\mathrm{H}}^{\prime}$ is the strength of the intermolecular hydrodynamic interaction, $c^{*}$ is the overlap concentration, and $R_{\mathrm{H}, 1}$ and $R_{\mathrm{H}, m 0}$ are hydrodynamic radii of unimer and $m_{0}$-mer, respectively. The overlap concentration may be calculated by $c^{*}=$ $3 M_{\mathrm{w}} / 4 \pi N_{\mathrm{A}} R_{\mathrm{H}}{ }^{3}$ where $N_{\mathrm{A}}$ is the Avogadro constant and $R_{\mathrm{H}}=M_{\mathrm{w}} /\left(w_{1} M_{1} R_{\mathrm{H}, 1}{ }^{-1}+w_{m 0} M_{m 0} R_{\mathrm{H}, m 0}{ }^{-1}\right)$. Using literature data of the hydrodynamic radius for linear PB in cyclohexane ${ }^{42}$ and of its $g$-factor for 4-arm star polymers in a good solvent $(0.93)^{26}$ as well as reasonable values for $k_{\mathrm{H}}^{\prime}$, we can draw the lines shown in Figure 2. The favorable fitting of $R_{\mathrm{H}, \mathrm{app}} / M_{\mathrm{w}}$ demonstrates that LPB exists as a four-arm star micelle in cyclohexane.

On the assumption that only free LPB (unimer) is the active species for the propagation reaction, the propagation rate is calculated by

$$
-\frac{d \ln \left([\mathrm{M}] /[\mathrm{M}]_{0}\right)}{d t}=k_{\mathrm{p}} w_{1} C_{0}
$$

where $[\mathrm{M}]$ and $[\mathrm{M}]_{0}$ are molar concentrations of the butadiene monomer at time $t$ and $t=0$, respectively, $C_{0}$ is the total molar concentration of LPB (i.e., the molar concentration of the initiator), $k_{\mathrm{p}}$ is the rate constant of the propagation reaction for free LPB, and $w_{1}$ is the weight fraction of the free LPB calculated from the free energy $\varepsilon_{0}$ of the micellar formation.

Figure 4 a compares literature propagation reaction rates of LPB in cyclohexane ${ }^{45,46}$ with eq (2.6) along with eqs (2.3) and (1.4) (and the normalization condition), using $\varepsilon_{0}=-14$, and $k_{\mathrm{p}}=133 \mathrm{M}^{-1} \mathrm{~s}^{-1}$. The reaction rate slightly depends on $M_{1}$ due to the non-ideality of the solution at higher LPB concentration or $C_{0}$. In Figure $4 \mathrm{a}$, we have calculated the theoretical reaction rate choosing $M_{1}=10^{4}$. As mentioned above, the value -14 was the upper limit of $\varepsilon_{0}$, and thus the $k_{\mathrm{P}}$ value $133 \mathrm{M}^{-1} \mathrm{~s}^{-1}$ chosen is its lower limit. It is noted that $k_{\mathrm{p}}$ of the free LPB is larger than that at the radical polymerization $\left(\approx 20 \mathrm{M}^{-1} \mathrm{~s}^{-1}\right),{ }^{47}$ and thus that the LPB anion is more reactive than the polybutadiene radical.

Recently, Oishi et $a .^{37}$ investigated the kinetics of living anionic polymerization for butadiene in deuterated benzene (a poor solvent for PB) by ${ }^{1} \mathrm{H}$ NMR. They observed nonsingle-exponential decays of the monomer concentration [M] during the propagation reaction, and the deviation from the single exponential decay was enhanced by addition of inert deuterated PB. This kinetic behavior may be explained by nonideality of the polymerization solution, as demonstrated in Figure $4 b$, where non-single-exponential curves were obtained from eqs (2.6), (1.4), and (2.3) with reasonable values of virial coefficients given in the caption of Figure $4 \mathrm{~b}$; the chain-end effect of the free LPB accounts for the positive $A_{11}$ in a poor solvent. ${ }^{48,49}$ The curves are favorably compared with Oishi et al.'s experimental results shown in Figures 4 and 9 in ref 37 .
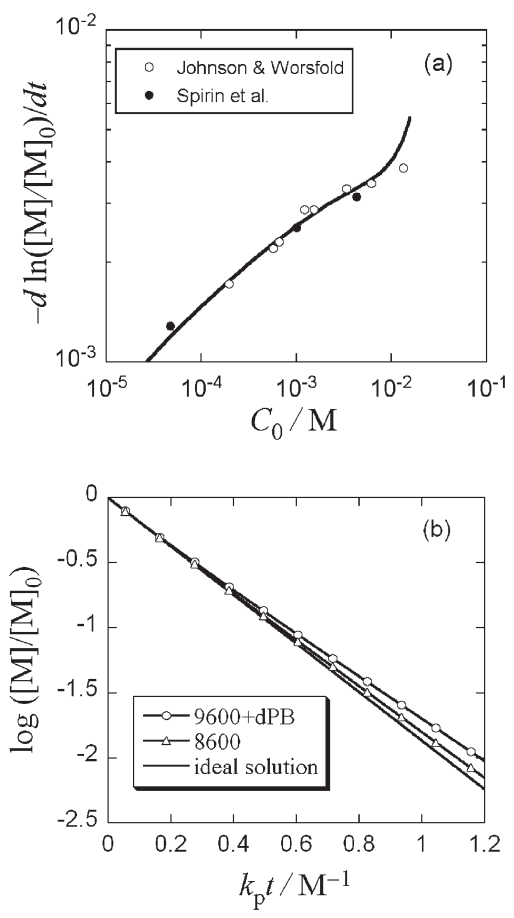

Figure 4. (a) Propagation reaction rate of the polybutadiene living anion in cyclohexane determined by Johnson and Worsfold ${ }^{46}$ and Spirin et al.; ${ }^{45}$ solid curve, theoretical values calculated by eq (2.6) along with eqs (1.4) and (2.3); (b) Non-single-exponential decay curves of butadiene monomer concentration [M] divided by the initial concentration $[\mathrm{M}]_{0}$ during propagation reaction of the living anionic polymerization, calculated with $w_{1}$ from eq (2.3) with $\varepsilon_{0}=-14, \quad A_{11}=3.5 \times 10^{-5} \mathrm{~cm}^{3} \mathrm{~mol} \mathrm{~g}^{-2}, \quad A_{44}=0, \quad A_{111}=5 \times$ $10^{-5} \mathrm{~cm}^{6} \mathrm{~mol} \mathrm{~g}^{-3}$, and $A_{444}=4 \times 10^{-5} \mathrm{~cm}^{6} \mathrm{~mol} \mathrm{~g}^{-3}$ under conditions of Batch codes $9(0)$ and $10(16,0.12)$ in ref 37 ; the ideal solution curve, obtained with all the virial coefficients to be zero.

\section{AMPHIPHILIC TELECHELIC POLYMER}

Amphiphilic telechelic polymers, i.e., hydrophilic polymer chains bearing hydrophobic groups on both ends, may be regarded as model polymers forming flower micelles in aqueous solution, where terminal hydrophobic groups are associated into a hydrophobic core and each hydrophilic polymer chain constructs a loop. ${ }^{10,13}$ With increasing the polymer concentration, however, such flower micelles may be interconnected by bridging chains with termini entrapped in the core of two distinct micelles, and exhibit the characteristic viscoelastic behavior. ${ }^{8,50-53}$ The association behavior of flower micelles is a delicate problem, because repulsive forces between loops tend to prevent micellar association whereas the loop-to-bridge transition by the entropic penalty associated with loop formation favors association. ${ }^{54,55}$

Recently, Nojima et al. ${ }^{9}$ investigated the association behavior of flower micelles formed by telechelic hydrophobicallymodified poly( $N$-isopropylacrylamide) (HM-PNIPAM) in $25^{\circ} \mathrm{C}$ water by static light scattering. The sample used was PNIPAM with octadecyl groups at both ends and the weightaverage molecular weight of 47,500.52,56,57 Water is a good solvent for PNIPAM at $25^{\circ} \mathrm{C}$ investigated. The weight-average 

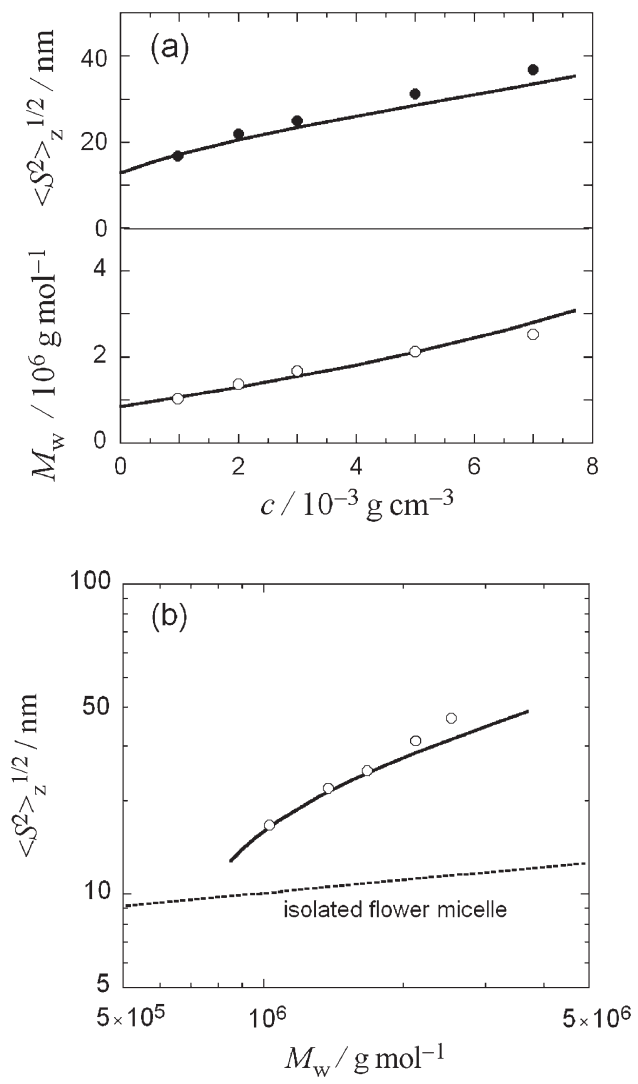

Figure 5. (a) Concentration dependences of the molar mass and the radius of gyration and (b) the molar mass dependence of the radius of gyration at different concentration for a telechelic HM-PNIPAM in $25^{\circ} \mathrm{C}$ water. Panel (b), reproduced with permission from ref 9 . Copyright (2008) American Chemical Society.

molar mass $M_{\mathrm{w}}$ and z-average square radius of gyration $\left\langle S^{2}\right\rangle_{\mathrm{z}}$ were determined as functions of the polymer concentration $c$ by static light scattering, where virial term corrections were made using theoretical second and third virial coefficients. ${ }^{9}$ Figure 5 displays their concentration dependences as well as the plot of $\left\langle S^{2}\right\rangle_{\mathrm{z}}{ }^{1 / 2}$ against $M_{\mathrm{w}}$. Both $M_{\mathrm{w}}$ and $\left\langle S^{2}\right\rangle_{\mathrm{z}}{ }^{1 / 2}$ sharply increase with $c$, and $\left\langle S^{2}\right\rangle_{\mathrm{z}}{ }^{1 / 2}$ exhibits a strong $M_{\mathrm{w}}$ dependence.

If telechelic HM-PNIPAM chains are assumed to form unicore flower micelles with an aggregation number $m$, the radius of gyration may be approximated to that of a star polymer which consists of $2 m$ arms of half PNIPAM chains. The radius of gyration $\left\langle S^{2}\right\rangle_{\text {star }}{ }^{1 / 2}$ of this star polymer may be calculated by eq $(1.12 \mathrm{~b})$ where $\left\langle S^{2}\right\rangle_{\text {linear }}$ for PNIPAM in $25^{\circ} \mathrm{C}$ water is empirically expressed by ${ }^{58}$

$$
\left\langle S^{2}\right\rangle_{\text {linear }}{ }^{1 / 2}=0.0188 \mathrm{~nm} \times M_{\mathrm{w}}{ }^{0.54}
$$

and $m$ is calculated from $M_{\mathrm{w}} / 47,500$. The dotted line in Figure $5 \mathrm{~b}$ indicates the result for $\left\langle S^{2}\right\rangle_{\mathrm{star}}{ }^{1 / 2}$. The experimental data points remarkably deviate from $\left\langle S^{2}\right\rangle_{\text {star }}{ }^{1 / 2}$ for all solutions and exhibit a much stronger molar mass dependence. This observation is a strong indication that the flower micelle association occurs in telechelic HM-PNIPAM solutions of $c$ as low as $1 \times 10^{-3} \mathrm{~g} / \mathrm{cm}^{3}$ by the bridging attraction due to the telechelic chain.
Then next, assuming that the flower micelle is regarded as an $f$-functional monomer with the molar mass $M_{\mathrm{u}}$ and the radius of gyration $\left\langle S^{2}\right\rangle_{\mathrm{u}}{ }^{1 / 2}$, we calculate $M_{\mathrm{w}}$ and $\left\langle S^{2}\right\rangle_{\mathrm{z}}{ }^{1 / 2}$ by using eqs (1.10), (1.11), and (1.22) as functions of $c$. In eq (1.11), we can calculate $F_{2}(\phi)$ by eq (1.3) with $\phi=c \bar{v}$ and the experimental partial specific volume $\bar{v}\left(=0.88 \mathrm{~cm}^{3} / \mathrm{g}\right)$ of PNIPAM in water. Extrapolating $M_{\mathrm{w}}$ and $\left\langle S^{2}\right\rangle_{\mathrm{z}}{ }^{1 / 2}$ to the zero $c$ in Figure $5 \mathrm{a}$, we can estimate $M_{\mathrm{u}}=8.5 \times 10^{5} \mathrm{~g} / \mathrm{mol}$ and $\left\langle S^{2}\right\rangle_{\mathrm{u}}{ }^{1 / 2}=12.8 \mathrm{~nm}$ for the unimer flower micelle, and the bond length $b$ between the nearest neighboring flower micelles approximately by the equation $b \approx 2 \times(5 / 3)^{1 / 2}\left\langle S^{2}\right\rangle_{\mathrm{u}}^{1 / 2}(=33$ $\mathrm{nm})$. Then the remaining unknown parameters in eqs (1.10), (1.11), and (1.22) are $f$ and $\Delta f_{0} / k_{\mathrm{B}} T$. The solid curve in Figure 5 indicates theoretical values calculated by these equations with $f=3$ and $\Delta f_{0} / k_{\mathrm{B}} T=-3.5$, which are in a fairly good agreement with experimental results. If we choose $f=4,{ }^{9}$ the fitting to the $c$ dependence of $M_{\mathrm{w}}$ becomes slightly worse. The theory seems to slightly underestimate $\left\langle S^{2}\right\rangle_{\mathrm{z}}{ }^{1 / 2}$ at high $c$ and $M_{\mathrm{w}}$, which may come from the intra-assembly excluded volume effect.

Equation (1.11) predicts the threshold concentration of gelation, corresponding to $\alpha=1 /(f-1)$, to be $0.024 \mathrm{~g} / \mathrm{cm}^{3}$. This prediction is consistent with Kujawa et al.'s report ${ }^{52}$ that aqueous solutions of a telechelic HM-PNIPAM with $M_{\mathrm{w}}=$ 37,000 formed gels above $c \sim 0.02 \mathrm{~g} / \mathrm{cm}^{3}$. (One may view each telechelic HM-PNIPAM chain as a bi-functional monomer and the hydrophobic core as multiple junction or crosslink point, instead of the unit flower micelle as the $f$-functional monomer. In such a view, however, the theory explained in the above THEORETICAL SECTION cannot be applied. ${ }^{59,60}$ )

\section{AMPHIPHILIC DIBLOCK COPOLYMER}

The micellization behavior of amphiphilic diblock copolymers in selective solvents has been investigated most extensively among associating polymers for many years, and a large amount of experimental and theoretical work has been accumulated so far. ${ }^{4,6,7,61-66}$ However, to our best knowledge, quantitative arguments have not thoroughly carried out yet on the detailed micellar structure using the radius of gyration and hydrodynamic radius obtained by light scattering.

Antonietti et $a l .{ }^{67}$ and subsequently Förster et al. ${ }^{68}$ extensively studied micelles of diblock copolymer consisting of polystyrene (PS) and poly(4-vinylpyridine) (P4VP) blocks in organic solvents. They prepared a number of PS- $b$-P4VP samples with different degrees of polymerization of PS $\left(N_{0, \text { core }}\right)$ and P4VP ( $\left.N_{0, \text { corona }}\right)$ blocks, and measured their molar masses $M_{\mathrm{w}}$ and light-scattering radii of gyration $\left\langle S^{2}\right\rangle^{* 1 / 2}$ of micelles formed in selective solvents by light scattering. (Since PS and P4VP are almost iso-refractive, $\left\langle S^{2}\right\rangle^{* 1 / 2}$ of their copolymers can be identified with true $\left\langle S^{2}\right\rangle^{1 / 2}$.) They focused on $N_{0, \text { core }}$ and $N_{0, \text { corona }}$ dependences of the aggregation number $m_{\mathrm{w}}$ calculated from $M_{\mathrm{w}}$, and obtained the semi-empirical scaling relation $^{68}$

$$
m_{\mathrm{w}} \propto N_{0, \text { core }}^{2} N_{0, \text { corona }}{ }^{-0.8}
$$




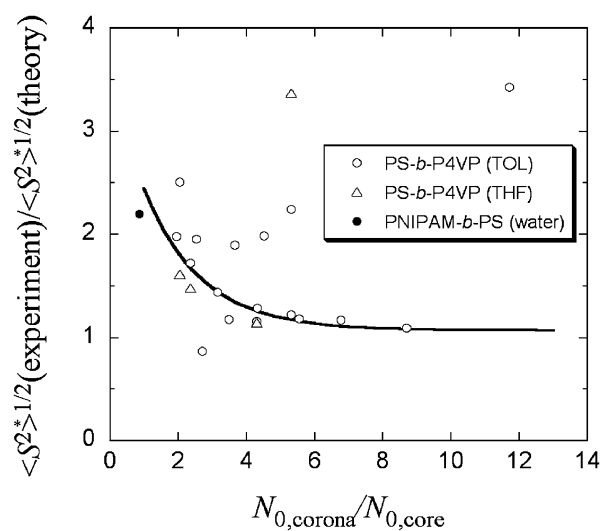

Figure 6. Comparison of experimental light-scattering radius of gyration $\left\langle S^{2}\right\rangle^{* 1 / 2}$ for amphiphilic block copolymers in selective solvents with the theoretical $\left\langle S^{2}\right\rangle^{* 1 / 2}$ for the core-corona micelle; solid curve, eye guide.

Here, we focus on their $\left\langle S^{2}\right\rangle^{* 1 / 2}$ data. Assuming that all PS$b$-P4VP samples they studied form core-corona micelles in toluene and THF (good solvents for PS), we calculate radii of gyration of their micelles using eq (1.16). Since we know the density $\rho_{\text {core }}$ of P4VP $\left(=1.05 \mathrm{~g} / \mathrm{cm}^{3}\right)$ and the molecular weight dependence of $\left\langle S^{2}\right\rangle_{\text {linear }}$ for PS in toluene and THF, ${ }^{69,70}$ we can calculate $\left\langle S^{2}\right\rangle^{* 1 / 2}$ for the core-corona micelle of each PS- $b$ P4VP sample with no adjustable parameters $\left(v_{\text {core }}=v_{\text {corona }}=\bar{v}\right.$ for PS- $b$-P4VP).

Figure 6 compares the theoretical $\left\langle S^{2}\right\rangle^{* 1 / 2}$ calculated by eq (1.16) with the experimental data for PS- $b$-P4VP in toluene and THF. Since eq (1.16) was derived on the basis of the segment density distribution for star polymers, it should be more accurate for core-corona micelles of smaller core sizes, or at larger $N_{0, \text { corona }} / N_{0 \text {,core }}$. In fact, ratios of the experimental to theoretical $\left\langle S^{2}\right\rangle^{* 1 / 2}$ seem to approach to unity at large $N_{0 \text {,corona }} / N_{0, \text { core }}$ in Figure 6 as shown by the eye guide curve. However, there are several points being appreciably deviated from unity in an irregular fashion, of which reasons are under consideration.

Recently, Zhang et al. ${ }^{71}$ investigated micelles of PNIPAM$b$-PS formed in water by transmission electron microscopy (TEM) and light scattering. From the TEM image, the authors judged that a PNIPAM- $b$-PS sample they investigated forms the vesicle in water. On the other hand, using eq (1.16), we can calculate $\left\langle S^{2}\right\rangle^{* 1 / 2}$ for the core-corona micelle formed by this PNIPAM- $b$-PS sample $\left(v_{\text {core }}=0.25 \mathrm{~cm}^{3} / \mathrm{g}^{72}\right.$ and $v_{\text {corona }}=$ $0.162 \mathrm{~cm}^{3} / \mathrm{g}^{58}$ ). As shown by the filled circle in Figure 6 , the deviation from unity of the ratio of the experimental $\left\langle S^{2}\right\rangle^{* 1 / 2}$ for the vesicle to the theoretical $\left\langle S^{2}\right\rangle^{* 1 / 2}$ for the core-corona micelle is comparable to the data of PS- $b$-P4VP micelles with similar $N_{0, \text { corona }} / N_{0, \text { core }}$.

We can also calculate $\left\langle S^{2}\right\rangle^{* 1 / 2}$ for the vesicle of Zhang et $a$.''s PNIPAM- $b$-PS sample $\left(N_{0, \text { shell }}=207 ; N_{0, \text { brush }}=176\right)$ by eq (1.22) along with eqs (1.18)-(1.21). These equations contain three adjustable parameters, $a, \kappa$, and $m_{\mathrm{i}} / m_{\mathrm{o}}$. Although it was difficult to determine those parameters uniquely, by choosing $a=1.9 \mathrm{~nm}, \kappa=0.0015$, and $m_{\mathrm{i}} / m_{\mathrm{o}}=0.3$ (i.e., $R=60 \mathrm{~nm}, \quad H_{\mathrm{i}}=18 \mathrm{~nm}$, and $H_{\mathrm{o}}=23 \mathrm{~nm}$ ) for example, eq (1.22) provides $\left\langle S^{2}\right\rangle^{* 1 / 2}$ in agreement with the experimental result. As expected, the brush heights $H_{\mathrm{i}}$ and $H_{\mathrm{o}}$ are larger than the average end-to-end distance $\left\langle R^{2}\right\rangle^{1 / 2}$ of the PNIPAM block chain in water $(=8.8 \mathrm{~nm})^{58}$ and smaller than $\left\langle R^{2}\right\rangle^{1 / 2}$ at the full extension $(=44 \mathrm{~nm})$. To increase the distance between neighboring brush chains, the PS block chain in the shell have to be compressed from the unperturbed state, so that the $a$ value considerably smaller than $\left\langle R^{2}\right\rangle^{1 / 2}$ of the PS chain in the $\theta$ state $(=10.7 \mathrm{~nm}),{ }^{73}$ is not unreasonable.

Eisenberg et $a l^{6,7}$ found various morphologies including vesicles, tubules, and lamellae for crew-cut aggregates of asymmetric block copolymers PS- $b$-poly(acrylic acid) with $N_{0, \text { corona }} / N_{0, \text { core }}<0.2$ by TEM. Iyama and Nose ${ }^{74}$ also reported vesicle and tubule formations by a PS- $b$-poly(dimethylsiloxane) sample with $N_{0 \text {,corona }} / N_{0 \text {,core }}=0.58$. Therefore, the possibility of bilayer morphologies should be checked for asymmetric block copolymers with smaller $N_{0 \text {,corona }} / N_{0 \text {,core }}$. Equation (1.16) is useful for this check.

\section{THERMALLY-DENATURED DOUBLE-HELICAL POLYSACCHARIDE}

Helical biopolymers (polysaccharides and proteins) are industrially important materials used as additives to foods, detergents, cosmetics, etc. to control their rheological properties. ${ }^{75-78}$ A microbial polysaccharide, xanthan, is one of such biopolymers with versatile properties in aqueous media and used in many areas of technology.

The main chain of xanthan is the same as cellulose and every other glucose residue possesses an ionic trisaccharide side chain. The native xanthan is known to take a double-helical structure and the double helix exhibits an order-disorder transition in aqueous solution by changing temperature and ionic strength. ${ }^{79-82}$ The disordered or denatured xanthan recovers its native circular dichroism (CD) by quenching and adding salt to the solution, and in what follows we call this phenomenon as the renaturation of xanthan. However, the viscosity of the renatured xanthan solution does not usually recover the native one, ${ }^{83}$ so that the local conformation of renatured xanthan is similar to that of the native but its global conformation is not necessarily identical with that of native xanthan. When the concentration of xanthan is high enough, the renatured xanthan solution even forms a gel, which is another important property of xanthan in industrial applications. ${ }^{84}$

Liu et al. ${ }^{85-87}$ carried out static light scattering to show that the double helix of xanthan was unwound from the ends, but did not dissociate into two dissociated single chains. Kawakami et $a l^{88}$ demonstrated by sedimentation equilibrium and viscometry that the denatured xanthan formed aggregates after renaturation, so that we can expect the mismatched winding of partially unwound double helices as illustrated in Figure $1 \mathrm{~h}$.

Recently, Matsuda et al. ${ }^{14}$ characterized aggregates of the renatured xanthan by size exclusion chromatography equipped with an online multi-angle light scattering detector (SECMALS). They prepared aqueous solutions of three xanthan samples listed in Table I with $c=1 \times 10^{-2} \mathrm{~g} / \mathrm{cm}^{3}$, and heated 
Table I. Molecular weights and polydispersities of native xanthan samples used for the renaturation experiments

\begin{tabular}{ccc}
\hline Sample & $M_{\mathrm{w}} / 10^{5}$ & $M_{\mathrm{w}} / M_{\mathrm{n}}$ \\
\hline S14 & 14 & 1.6 \\
S5 & 5.1 & 1.1 \\
S1 & 1.2 & $<1.1$
\end{tabular}

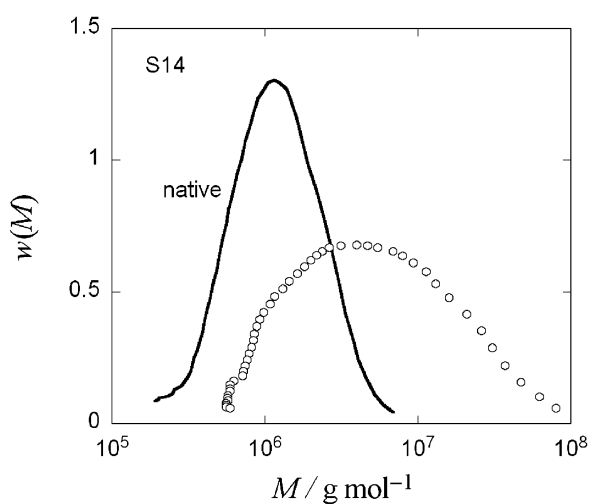

Figure 7. Molar mass distributions $w(M)$ of native and renatured sample S14, obtained by SEC-MALS.

the solutions at $80^{\circ} \mathrm{C}$ for $30 \mathrm{~min}$. Aqueous $\mathrm{NaCl}$ was added to the denatured xanthan solutions to adjust the concentrations of xanthan and $\mathrm{NaCl}$ to $5 \times 10^{-3} \mathrm{~g} / \mathrm{cm}^{3}$ and $0.1 \mathrm{M}$, respectively. Those solutions were left at the room temperature for $24 \mathrm{~h}$ after the addition of $\mathrm{NaCl}$, and then further diluted by $0.1 \mathrm{M}$ aqueous $\mathrm{NaCl}$ to be used for SEC-MALS measurements. The aggregation was stopped by this dilution. Circular dichroism demonstrated that the renatured xanthan recovered locally the original double helical structure.

Figure 7 compares the molar mass distribution of a xanthan sample S14 after renaturation with that of the native sample, where $w(M)$ is the weight fraction of the xanthan component with the molar mass $M$, obtained by SEC-MALS. The distribution curve of the renatured sample shifts to higher $M$ region, and becomes broader than that of native one, demonstrating the aggregation of the denatured xanthan dimer.

Figure 8 shows the molar mass $M$ dependences of the radius of gyration $\left\langle S^{2}\right\rangle^{1 / 2}$ obtained by SEC-MALS for fractions of the renatured xanthan samples S14, S5, and S1. Data points for all the fractions do not agree with that for the native xanthan double helix indicated by the dot-dashed curve, demonstrating that the renatured samples do not recover the original double helix. This is in a contrast with the CD result almost recovering the native spectrum after renaturation.

From the above SEC-MALS and CD results, we can imagine that denatured xanthan dimers form aggregates by the mismatched winding as illustrated in Figure 1h. This aggregation can be viewed as the polycondensation of monomers indicated by circles in Figure 9, i.e., $m$ partially unwound xanthan double helices form $(m+1)$-mer. The monomers shown in Figure 9 are bi-functional $(f=2)$ or tri-functional $(f=3)$. We denote the contour lengths of the intact double

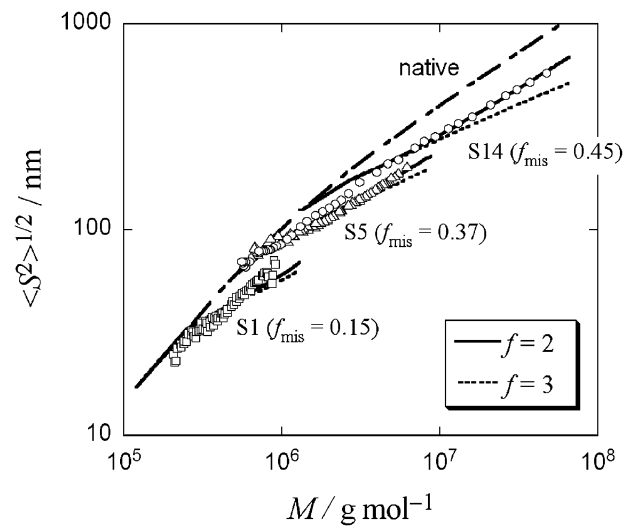

Figure 8. Molar mass dependences of $\left\langle S^{2}\right\rangle^{1 / 2}$ for three renatured xanthan samples, S14, S5, and S1, with different molecular weights.

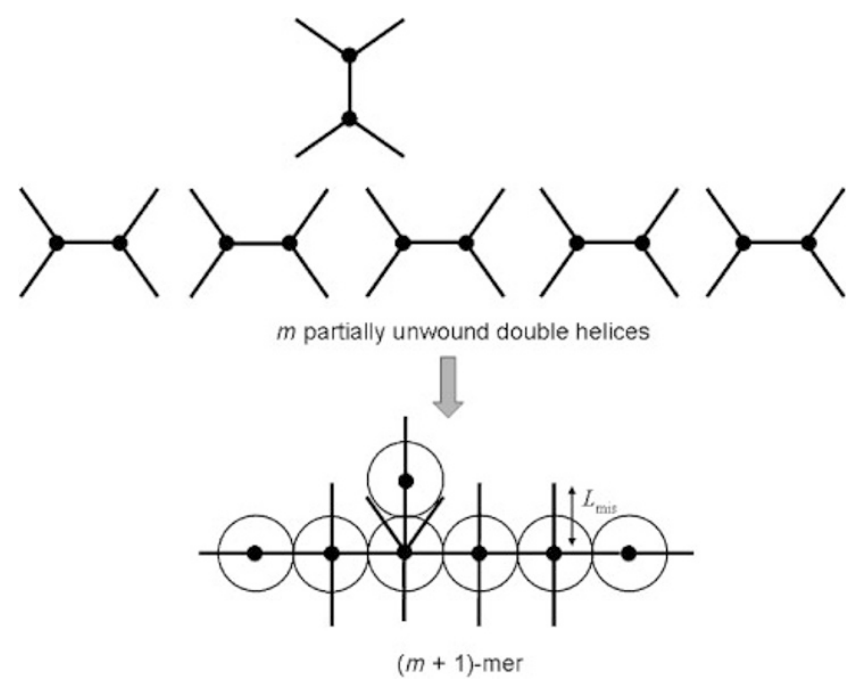

Figure 9. Schematic illustrate of a xanthan aggregate formed from $\mathrm{m}$ partially unwound double helices.

helix and mismatched double helix portion as $L_{1}$ and $L_{\text {mis }}$, respectively. The square radius of gyration of the $m$-mer can be given by eq (1.25). In the equation, $b$ and $\left\langle S^{2}\right\rangle_{\mathrm{u}}$ may be calculated by

$$
b^{2}=\left\langle R^{2}\right\rangle\left(\left(1-f_{\text {mis }}\right) N_{1}\right), \quad\left\langle S^{2}\right\rangle_{\mathrm{u}}=\left\langle S^{2}\right\rangle\left(f_{\text {mis }} N_{1}\right)
$$

where $\left\langle R^{2}\right\rangle(N)$ and $\left\langle S^{2}\right\rangle(N)$ are the mean square end-to-end distance and the mean square radius of gyration of the wormlike chain, respectively, as a function of the Kuhn statistical segment number $N, f_{\text {mis }}$ is the mismatch fraction ( $\equiv 2 L_{\text {mis }} / L_{1}$ ), and $N_{1}$ is the Kuhn statistical segment number of the native xanthan sample ( $\equiv L_{1} / 2 q ; q$ : the persistence length). Kratky and Porod ${ }^{89}$ and Benoit and Doty ${ }^{90}$ presented $\left\langle R^{2}\right\rangle(N)$ and $\left\langle S^{2}\right\rangle(N)$, respectively, for the wormlike chain model in the forms

$$
\begin{aligned}
& \left\langle R^{2}\right\rangle(N)=(2 q)^{2}\left[N-\frac{1}{2}\left(1-\mathrm{e}^{-2 N}\right)\right] \\
& \left\langle S^{2}\right\rangle(N)=(2 q)^{2}\left\{\frac{1}{6} N-\frac{1}{4}+\frac{1}{4 N}\left[1-\frac{1}{2 N}\left(1-\mathrm{e}^{-2 N}\right)\right]\right\}
\end{aligned}
$$


Solid and dotted curves in Figure 8 indicate theoretical values calculated by the polycondensate model for $f=2$ and 3 , respectively. Here, $N_{1}$ was calculated from $M_{\mathrm{w}}$ for each sample ( $c f$. Table I) with $M_{\mathrm{L}}=1940 \mathrm{~nm}^{-1}$ and $q=100 \mathrm{~nm}$, by neglecting polydispersity in the molecular weight. For all xanthan samples, experimental data points are almost fitted to the solid curve with $f_{\text {mis }}$ indicated in the figure. The mismatch fraction $f_{\text {mis }}$ chosen increases with $M_{\mathrm{w}}$ of the sample. The fitting is slightly worse if one chooses $f=3$. The junction for $f=3$ must be formed only at simultaneous gathering of three denatured xanthan dimers which may seldom occur in relatively dilute solution.

\section{SUMMARY}

Light scattering studies on the following macromolecular assemblies were overviewed:

(1) Polybutadiene living anions in cyclohexane. The aggregation number and the lower limit of the association constant were determined by light scattering. From these results, the lower limit of the true propagation rate constant in the living anionic polymerization of butadiene was estimated.

(2) Telechelic hydrophobically-modified poly( $N$-isopropylacrylamide) in water. The association behavior of flower micelles through the bridging attraction was examined by light scattering. The free energy of the loop/bridge exchange estimated from light scattering data predicted reasonable gelation threshold.

(3) Amphiphilic block copolymers in selective solvents. Previously reported experimental radii of gyration of block copolymer micelles were compared with theoretical ones calculated for the core-corona micelle model. Not all data points were favorably compared with the theoretical, demonstrating that the micellar structure formed by diblock copolymers in selective solvents has not fully understood.

(4) Thermally denatured double-helical polysaccharide xanthan after renaturation. The aggregation number distribution and the assembly structure of the renatured xanthan were investigated by size-exclusion chromatography equipped with an online multi-angle light scattering detector. The experimental results obtained were favorably compared with the model of the mismatched winding among partially unwound double helices.

Received: September 30, 2008 Accepted: November 19, 2008 Published: January 15, 2009

\section{REFERENCES}

1. "Supramolecular Polymers," A. Ciferri, Ed., Marcel Dekker, New York and Basel, 2000.

2. L. Zhang and A. Eisenberg, Science, 268, 1728 (1995).

3. L. Zhang, K. Yu, and A. Eisenberg, Science, 272, 1777 (1996).

4. K. Yu and A. Eisenberg, Macromolecules, 31, 3509 (1998).

5. K. Iyama and T. Nose, Polymer, 39, 651 (1998).

6. H. Shen and A. Eisenberg, Macromolecules, 33, 2561 (2000).
7. D. E. Discher and A. Eisenberg, Science, 297, 967 (2002).

8. B. Xu, L. Li, A. Yekta, Z. Masoumi, S. Kanagalingam, M. A. Winnik, K. Zhang, P. M. Macdonald, and S. Menchen, Langmuir, 13, 2447 (1997).

9. R. Nojima, T. Sato, X. Qiu, and F. M. Winnik, Macromolecules, 41, 292 (2008)

10. A. N. Semenov, J.-F. Joanny, and A. R. Khokhlov, Macromolecules, 28, 1066 (1995).

11. O. V. Borisov and A. Halperin, Langmuir, 11, 2911 (1995).

12. O. V. Borisov and A. Halperin, Macromolecules, 29, 2612 (1996).

13. A. Halperin, "Supramolecular Polymers," A. Ciferri, Ed., Marcel Dekker, New York and Basel, 2000, p. 93.

14. Y. Matsuda, Y. Biyajima, and T. Sato, Polym. J., submitted (2008).

15. T. Kimura, A. Hashidzume, and T. Sato, Prog. Theor. Phys., in press (2008).

16. F. Tanaka, Macromolecules, 22, 1988 (1989).

17. T. Sato, Langmuir, 20, 1095 (2004).

18. P. J. Flory, "Principles of Polymer Chemistry," Cornell Univ. Press, Ithaca, New York, 1953.

19. T. Sato, Y. Jinbo, and A. Teramoto, Macromolecules, 30, 590 (1997).

20. J. N. Israelachvili, "Intermolecular and Surface Forces," 2nd ed, Academic Press, London, 1992.

21. Y. Matsuda, R. Nojima, T. Sato, and H. Watanabe, Macromolecules, 40, 1631 (2007).

22. W. H. Stockmayer, J. Chem. Phys., 11, 45 (1943).

23. B. H. Zimm and W. H. Stockmayer, J. Chem. Phys., 17, 1301 (1949).

24. A. J. Barrett and D. L. Tremain, Macromolecules, 20, 1687 (1987).

25. K. Shida, K. Ohno, M. Kimura, Y. Kawazoe, and Y. Nakamura, Macromolecules, 31, 2343 (1998).

26. J. F. Douglas, J. Roovers, and K. F. Freed, Macromolecules, 23, 4168 (1990).

27. M. Daoud and J. P. Cotton, J. Phys. (Paris), 43, 531 (1982).

28. H. Yamakawa, "Modern Theory of Polymer Solutions," S. A. Rice, Ed., Harper \& Row, New York, 1971.

29. H. Benoit and D. Froelich, in "Light Scattering from Polymer Solutions," M. B. Huglin, Ed., Academic Press, London \& New York, 1972, vol. 27, p. 467.

30. S. T. Milner, T. A. Witten, and M. E. Cates, Macromolecules, 21, 2610 (1988).

31. G. R. Dobson and M. Gordon, J. Chem. Phys., 41, 2389 (1964).

32. K. Kajiwara, Polymer, 12, 57 (1971).

33. H. L. Hsieh and R. P. Quirk, "Anionic Polymerization," Marcel Dekker, New York, 1996.

34. R. N. Young, R. P. Quirk, and L. J. Fetters, Adv. Polym. Sci., 56, 1 (1984).

35. M. van Beylen, S. Bywater, G. Smets, M. Szwarc, and D. J. Worsfold, Adv. Polym. Sci., 86, 87 (1988).

36. K. Yamauchi, H. Hasegawa, T. Hashimoto, H. Tanaka, R. Motokawa, and S. Koizumi, Macromolecules, 39, 4531 (2006).

37. Y. Oishi, Y. Matsumiya, and H. Watanabe, Polym. J., 39, 304 (2007).

38. Y. Matsuda, T. Sato, Y. Oishi, and H. Watanabe, J. Polym. Sci., Part B: Polym. Phys., 43, 1401 (2005).

39. M. Okumoto, Y. Nakamura, T. Norisuye, and A. Teramoto, Macromolecules, 31, 1615 (1998).

40. M. Okumoto, Y. Iwamoto, Y. Nakamura, and T. Norisuye, Polym. J., 32, 422 (2000).

41. T. Norisuye and H. Fujita, Chemtracts: Macromol. Chem., 2, 293 (1991).

42. J. Roovers and J. E. Martin, J. Polym. Sci., Part B: Polym. Phys., 27, 2513 (1989).

43. T. Kanematsu, T. Sato, Y. Imai, K. Ute, and T. Kitayama, Polym. J., 37, 65 (2005).

44. R. Nojima, A. Hashidzume, and T. Sato, Macromol. Symp., 249-250, 502 (2007).

45. Y. L. Spirin, A. R. Gantmakher, and S. S. Medvedev, Dokl. Akad. Nauk. SSSR, 146, 368 (1962).

46. A. F. Johnson and D. J. Worsfold, J. Polym. Sci., A, 3, 449 (1965). 
47. F. Bandermann, C. Günter, and J. Schweer, Macromol. Chem. Phys., 197, 1055 (1996).

48. H. Yamakawa, Macromolecules, 25, 1912 (1992).

49. Y. Einaga, F. Abe, and H. Yamakawa, Macromolecules, 26, 6243 (1993).

50. E. Alami, M. Almgren, W. Brown, and J. François, Macromolecules, 29, 2229 (1996).

51. T. Annable, R. Buscall, R. Ettelaie, and D. Whittlestone, J. Rheol., 37, 695 (1993)

52. P. Kujawa, H. Watanabe, F. Tanaka, and F. M. Winnik, Eur. Phys. J. E, 17, 129 (2005).

53. F. Tanaka and T. Koga, Macromolecules, 39, 5913 (2006).

54. G. ten Brinket and G. Hadziioannou, Macromolecules, 20, 486 (1987).

55. N. P. Balsara, M. Tirrell, and T. P. Lodge, Macromolecules, 24, 1975 (1991).

56. P. Kujawa, F. Segui, S. Shaban, C. Diab, Y. Okada, F. Tanaka, and F. M. Winnik, Macromolecules, 39, 341 (2006).

57. P. Kujawa, F. Tanaka, and F. M. Winnik, Macromolecules, 39, 3048 (2006).

58. K. Kubota, S. Fujishige, and I. Ando, Polym. J., 22, 15 (1990).

59. K. Fukui and T. Yamabe, Bull. Chem. Soc. Jpn., 40, 2052 (1967).

60. F. Tanaka and W. H. Stockmayer, Macromolecules, 27, 3943 (1994).

61. A. Qin, M. Tian, C. Ramireddy, S. E. Webber, P. Munk, and Z. Tuzar, Macromolecules, 27, 120 (1994).

62. B. Chu, Langmuir, 11, 414 (1995).

63. S. Förster and M. Antonietti, Adv. Mater., 10, 195 (1998).

64. M. Antonietti and S. Förster, Adv. Mater., 15, 1323 (2003).

65. S. Förster, V. Abetz, and A. H. E. Müller, Adv. Polym. Sci., 166, 173 (2004).

66. Y. Morishima, Angew. Chem., Int. Ed., 46, 1370 (2007).

67. M. Antonietti, S. Heinz, M. Schmidt, and C. Rosenauer, Macromolecules, 27, 3276 (1994).

68. S. Förster, M. Zisenis, E. Wenz, and M. Antonietti, J. Chem. Phys., 104, 9956 (1996).
69. F. Abe, Y. Einaga, T. Yoshizaki, and H. Yamakawa, Macromolecules, 26, 1884 (1993).

70. "Polymer Handbook," J. Brandrup and E. H. Immergut, Ed., 3rd ed, John Wiley \& Sons, New York, 1989.

71. W. Zhang, X. Zhou, H. Li, Y. Fang, and G. Zhang, Macromolecules, 38, 909 (2005).

72. "Light Scattering from Polymer Solutions," M. B. Huglin, Ed., Academic Press, London \& New York, 1972.

73. T. Konishi, T. Yoshizaki, T. Saito, Y. Einaga, and H. Yamakawa, Macromolecules, 23, 290 (1990).

74. K. Iyama and T. Nose, Macromolecules, 31, 7356 (1998).

75. A. H. Clark and S. B. Ross-Murphy, Adv. Polym. Sci., 83, 57 (1987).

76. J.-M. Guenet, "Thermoreversible Gelation of Polymers and Biopolymers," Academic Press, London, 1992.

77. K. te Nijenhuis, Adv. Polym. Sci., 130, 1 (1997).

78. F. Tanaka, Macromolecules, 36, 5392 (2003).

79. T. Sato, T. Norisuye, and H. Fujita, Polym. J., 16, 341 (1984).

80. T. Sato, S. Kojima, T. Norisuye, and H. Fujita, Polym. J., 16, 423 (1984).

81. T. Sato, T. Norisuye, and H. Fujita, Macromolecules, 17, 2696 (1984).

82. T. Norisuye and A. Teramoto, in "Polymeric Materials Encyclopedia," J. C. Salamone, Ed., CRC Press, Boca Raton, 1996, vol. 11, p. 8801

83. H. W. Oviatt and D. A. Brant, Macromolecules, 27, 2402 (1994).

84. S. B. Ross-Murphy, V. J. Morris, and E. R. Morris, Faraday Symp. Chem. Soc., 18, 115 (1983).

85. W. Liu, T. Sato, T. Norisuye, and H. Fujita, Carbohydr. Res., 160, 267 (1987).

86. W. Liu and T. Norisuye, Biopolymers, 27, 1641 (1988).

87. W. Liu and T. Norisuye, Int. J. Biol. Macromol., 10, 44 (1988).

88. K. Kawakami, Y. Okabe, and T. Norisuye, Carbohydr. Polym., 14, 189 (1991).

89. O. Kratky and G. Porod, Rec. Trav. Chem., 68, 1106 (1949).

90. H. Benoit and P. Doty, J. Phys. Chem., 57, 958 (1953).

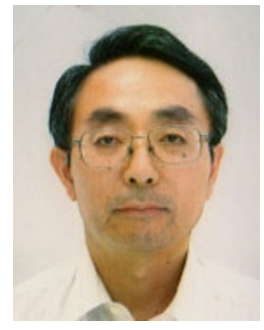

Takahiro SATO was born in Osaka, Japan, in 1957, graduated from the Department of Macromolecular Science, Osaka University in 1980, and received his PhD from Osaka University in 1985. He started working in 1985 as a guest scientist at National Bureau of Standards (now National Institute of Standards and Technology) in USA, and then joined the Department of Macromolecular Science, Osaka University as an assistant professor in 1987. He promoted to an associate professor in 1996 and a full professor in 2002 at Osaka University. He received the Award of the Society of Polymer Science, Japan in 2008. His major research field is polymer solution properties, and he is interested in interrelations among the conformation, interaction, higher-order structure, and various solution properties of stiff-chain polymers, helical polymers, and associating polymers.

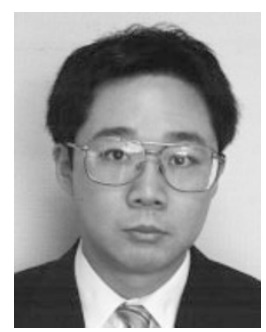

Yasuhiro MATSUDA was born in Shizuoka, Japan in 1979, graduated from Department of Chemistry, Osaka University in 2002, and received his PhD from Osaka University in 2006. He started working in 2006 as a postdoctoral fellow at Institute for Materials Chemistry and Engineering, Kyushu University, and then joined the Department of Materials Science and Chemical Engineering, Shizuoka University as an assistant professor in 2007. His present research interest is relations between solution properties and surface and interfacial properties of various polymers. 